\title{
La malaria en el Amazonas: conocimientos, prácticas, prevalencia de parasitemia y evaluación entomológica en mayo de 1997
}

\author{
Ligia Pérez ${ }^{1}$ Martha Suárez², Luz Murcia ${ }^{3}$, Fernando De la Hoz ${ }^{4}$, \\ Victor Alberto Olano ${ }^{5}$, Helena Brochero ${ }^{5}$ Pedro Toro 6
}

\begin{abstract}
Resumen
Se realizó un estudio epidemiológico de malaria en el departamento del Amazonas que incluía la medición de los conocimientos y prácticas sobre malaria que tenían los pobladores de Arica en el río Putumayo, la prevalencia de parasitemia en una muestra de personas que viven en el área y el análisis de los datos de la vigilancia epidemiológica del departamento durante 1996. En el estudio de grupos focales en Arica, se encontró que las personas identifican a la malaria como una enfermedad transmitida por un mosquito, pero también, hay una proporción de personas que piensan que es transmitida por beber agua contaminada. La prevalencia de parasitemia en 37 muestras tomadas de personas que habían tenido enfermedad febril recientemente fue de $8 \%$. Las personas que trabajaban en cultivos de coca tenían la mayor parasitemia $(27 \%)$, mientras que ninguno de los que no se dedicaban a esta actividad era positivo, $p<0,01$. En el grupo focal, la mayoría estuvo de acuerdo en que la enfermedad era producida por un mosquito, pero, en la encuesta, una proporción apreciable de personas dio respuestas equivocadas. Como posible vector de malaria en el área, solamente se encontró Anopheles oswaldoi, mientras que no se identificó Anopheles darlingi. Se notificaron 307 casos de malaria en el departamento durante 1996, de los cuales 72 fueron causados por Plasmodium falciparum (24\%) y el resto por $P$. vivax. De 10 localidades analizadas, solamente en una predominaba P. falciparum (La Chorrera), mientras que la localidad de Tarapacá reportó casos todo el año. El estudio muestra que la mayor parte del departamento del Amazonas es de transmisión inestable para malaria, pero hay por lo menos una localidad donde la transmisión es permanente, Tarapacá. También, se logró identificar a los trabajadores de cultivos ilícitos como una población de alto riesgo para el mantenimiento de la circulación del parásito en estas poblaciones.
\end{abstract}

Palabras clave: malaria, factores de riesgo y encuestas CAP

1 Control de Vectores, Secretaría de Salud del Amazonas, Residente SEA/INS.

2 Coordinación de Epidemiología, Secretaría de Salud del Amazonas

3 Laboratorio Departamental de Salud Pública, Departamento del Amazonas

4 Centro Control de Enfermedades Transmisibles, División de Centros de Control de Enfermedades, INS.

5 Laboratorio de Entomología, Laboratorio Nacional de Referencia, INS

6 Grupo de Control de vectores, Secretaría de Salud del Amazonas.

Recibido febrero 28 de 1999, aceptado junio 18 de 1999 


\title{
Malaria in the Amazon region: knowledge, practices, prevalence of parasitaemia and entomological evaluation in May, 1997
}

\begin{abstract}
A study of malaria epidemiology was carried out in the Amazonas Department (Colombia). The study's aims were to measure the state of knowledge and practice regarding malaria frequency and control techniques among people living in Arica (a village on the banks of the Putumayo river) and to evaluate the prevalence of positive malaria blood smears taken from people living in this village. Malaria surveillance data for the Amazonas (i.e. that available for 1996) was also analysed. Using focus groups, it was found that most people identify malaria as being a mosquito-borne disease, but, in a house-tohouse survey made after the focus groups were held, an important proportion of people also believe that contaminated water could be a potential source of infection. $8 \%$ of people presenting recent febrile illness were positive for malaria (blood smear). Those people involved in activities related to coca growing and collection had a higher prevalence $(27 \%)$ while those who were not involved in such activities were all negative for malaria, $p<0.001$. Anopheles darlingi was not identified in this area, despite the fact that it is the main malarial vector in other areas of the Amazonas. Anopheles oswaldoi was the only potential malarial vector detected in these villages. Overall, 307 cases of malaria were detected by the surveillance system in 1996 throughout the department. Most cases were caused by Plasmodium vivax (76\%); Plasmodium falciparum was found in $24 \%$ of the cases. In Tarapacá cases of malaria were detected during each month throughout 1996 while in other villages there were some periods having no reported cases.

This study shows that malaria transmission is unstable in the Amazonas department excepting Tarapacá where transmission is permanent. We can support that most cases are caused by Plasmodium vivax while an important proportion of people living in risk areas is unaware of how the disease is transmitted. Also, we found that people involved in coca growing in the jungle have the highest risk for malaria and they can be an important factor in the maintenance of malaria in these communities.
\end{abstract}

Key words: malaria, risk factors, KAP surveys

\section{Introducción}

Los cultivos ilícitos, el orden público, las migraciones, la colonización, la población dispersa y las zonas fronterizas se han identificado como los principales factores que intervienen en la transmisión de la malaria en la Amazonia colombiana (1).

El departamento del Amazonas presenta condiciones ecológicas excepcionales para que se encuentren presentes en su territorio una variedad de especies de anofelinos Además de los aspectos ecológicos, también ha sido escenario de una serie de cambios sociales (inmigración de población para explotación maderera y para cultivo y procesamiento de coca) que se han convertido en factores que propician la presentación de casos y brotes de malaria. Tradicionalmente, los focos de casos de malaria se han presentado en el corregimiento de Tarapacá, en donde se concentra una actividad importante de explotación maderera; sin embargo, en los últimos años hay registros de casos en localidades donde no estaba documentada la transmisión, como en los corregimientos de Arica y Puerto Limón. Durante 1995, la localidad de Arica reportó seis casos de malaria hasta la semana epidemiológica 12, lo cual es un incremento estadísticamente significativo con respecto a los casos esperados según los años anteriores.

Dadas estas condiciones, la Secretaría de Salud del Amazonas realizó bajo la asesoría del Instituto 
Nacional de Salud, una evaluación sobre la situación de malaria en el departamento y una investigación de campo sobre los factores asociados con la enfermedad y las creencias y prácticas sobre malaria de los habitantes de la zona. Con este propósito, se decidió realizar un análisis epidemiológico de la situación de la malaria en el departamento del Amazonas durante 1996 y 1997 y caracterizar la información según las variables de persona, tiempo y lugar, medir la prevalencia de conocimientos y prácticas de prevención sobre la malaria en las poblaciones de Arica y Puerto Limón, estimar la prevalencia de malaria en la población de Arica en el período 1995-1997, estimar la prevalencia de parasitemia en una muestra de habitantes de esta región, recolectar e identificar taxonómicamente las especies de anofelinos adultos, caracterizar los criaderos potenciales de Anophelesy determinar las posibles medidas de control larvario.

\section{Materiales y métodos}

Las actividades de campo se llevaron a cabo en Arica y Puerto Limón. Para evaluar los conocimientos y las prácticas sobre malaria, se acudió a la metodología de los grupos focales. A través del promotor y de los líderes se convocó a la comunidad ubicada en la cabecera del corregimiento de Arica a una reunión con el grupo de trabajo; se organizaron grupos focales de discusión sobre malaria. Siguiendo las recomendaciones de la OMS para la conducción de estos grupos, se dividieron en dos grupos de no más de 10 personas cada uno y cada uno de los grupos estuvo conformado por personas de un solo género, hombres o mujeres. Participaron 17 personas en los grupos focales, uno estaba compuesto de 9 mujeres y el otro por 8 hombres; todos eran mayores de 25 años.

El grupo de investigadores elaboró previamente una guía de discusión. Esta guía contenía los siguientes puntos: conocimientos de la comunidad sobre la malaria y el paludismo -los nombres que reciben en la comunidad-; la percepción que tiene la comunidad sobre el origen y las formas de transmisión; la importancia que se les da a los mosquitos como agentes de enfermedades, especialmente malaria; las formas de protección que la comunidad adopta para su protección; el acceso a los mecanismos de control individual y colectivo, y la impresión que tienen sobre las actividades de control de la malaria que el Estado desarrolla.

Posteriormente, se elaboró una encuesta sobre prevalencia de enfermedad similar a la malaria durante los dos últimos años y sobre la prevalencia y uso de toldillos. La encuesta fue probada en una muestra de la población de la cabecera del corregimiento de Arica; una vez corregida, se aplicó a los jefes de familia en las poblaciones de Arica y Puerto Limón.

Se tomaron muestras de gota gruesa a todos los individuos de Arica que refirieron haber tenido malaria en los últimos tres años y en Puerto Limón, se les tomó a todos los habitantes de una plantación de coca que estaba situada a media hora de allí. La bacterióloga y el promotor de salud de Arica, quienes están entrenados en microscopía para malaria, examinaron en campo las muestras de gota gruesa; las personas que resultaron positivas para Plasmodium recibieron tratamiento antimalárico. Se comparó la frecuencia de resultados positivos en las dos localidades, usando una prueba de chi cuadrado con un nivel de confianza de $95 \%$.

Simultáneamente, se realizó una inspección de criaderos potenciales y captura de Anopheles; se consideraron como criaderos potenciales los caños represados, las quebradas represadas, las charcas de agua lluvia y los chuquios. Los chuquios localizados en las zonas selváticas son depresiones del terreno que acumulan agua de lluvia y hojarasca. Para la captura de Anopheles adultos, se utilizó trampa Shannon, trampa CDC (luz) y cebo humano protegido; la captura se realizó entre las 18 y las 20 horas.

Se analizó la información de la vigilancia epidemiológica sobre los casos de malaria que consultan a los centros de salud del departamento, la cual está sistematizada en una base de datos de Epiinfo 6.04 en la Secretaría de Salud del Amazonas. Esta información fue analizada y se calcularon las frecuencias de positivos por grupos de edad y sexo; se calcularon las tasas de incidencia ajustadas por edad para 
cada una de las localidades. Se analizó, también, la incidencia por semanas epidemiológicas para las localidades más importantes.

\section{Resultados}

Conocimientos y prácticas sobre la malaria y su control: se encuestaron 63 personas, 32 hombres y 31 mujeres; $43(68,3 \%)$ residentes en Arica, 10 $(15,9 \%)$ en Puerto Limón, 8 (12,7\%) ubicados en las afueras de Puerto Limón y $2(12,7 \%)$ en Buri Buri; $44 \%$ pertenecía a la etnia Huitoto, 20,6\% de la etnia Bora, $19 \%$ colonos, $12,7 \%$ peruanos y $3,2 \%$ de las etnias Ingano de Ecuador y Miraña en la zona del Amazonas. En Arica, las personas entrevistadas pertenecen a las etnias Huitoto y Bora, mientras que, en Puerto Limón, había un mayor porcentaje de colonos y peruanos.

Conocimientos de mosquitos: las comunidades estudiadas identifican varias clases de mosquitos por sus nombres indígenas, pero, en general, no pueden distinguir claramente las características del Anopheles de las de otros zancudos, ni cual de ellos puede ser el vector de la malaria.

Algunos de los nombres usados y las características de los mosquitos que reciben ese nombre son las siguientes:

Marudo: mosquito largo y delgado que pica en el monte y en la chagra y, según ellos, no creen que sea el transmisor de la malaria.

Ako: un mosquito grande de color moreno que vive en el peridomicilio y también pica.

Ikenakwaje: un mosquito de color amarillo, pequeño, que vive en el monte y que no pica.

Namejawaji: mosquitos caseros que creen que salen de las letrinas y de las partes oscuras de la casa; son nocturnos y también son muy molestos por su picadura.

Kuaki ipa (pito): mosquito grande, cenizo, cuya picadura produce tumor y llaga.

Wuinnita: un mosquito pequeño, blanco, que pica en el domicilio por la noche y produce lesiones en la piel como ronchas.

Sobre el comportamiento de los mosquitos, la gente opina que son más abundantes cuando el río está en fase de crecimiento y pica más por las tardes y en las afueras de las casas.
Conocimientos sobre las enfermedades transmitidas por el mosquito: las personas saben que la malaria puede ser transmitida por un mosquito, debido a la información que les han dado los trabajadores del Grupo de Control de Vectores y los del puesto de salud, aunque también identifican otras fuentes incorrectas de infección por malaria, como el beber agua del río y de las charcas estancadas en la selva. Las personas entrevistadas referían que la mayor necesidad de control sobre los mosquitos se debía a que eran causa de molestia en la vivienda por su picadura y su zumbido por las noches; ninguno identificaba la transmisión de malaria con las necesidades de control de los zancudos. Unos pocos entrevistados mencionaron que el nuche podía ser también transmitido por un mosquito. No se mencionaron enfermedades como la leishmaniasis, el dengue o la fiebre amarilla, como transmitidas por zancudos.

Sitios de cría de los mosquitos: identifican como principales fuentes de cría de zancudos, las charcas y los depósitos de agua fuera de la vivienda, en el peridomicilio o en el campo, pero no dentro de ella; identificaron que a los zancudos les gusta descansar, principalmente en el peridomicilio y en los sitios oscuros dentro de la vivienda.

Medidas de protección personal: la mayoría de las personas considera que es necesario protegerse de la picadura de los mosquitos y sobre todo de su molesto zumbido por las noches; todos coincidieron en que la medida más efectiva de protección era el uso de toldillos por las noches. Todos los asistentes afirmaron tener toldillos en sus casas y tener uno para cada uno de los habitantes de la casa. Esto es especialmente importante, si se tiene en cuenta que el valor de un toldillo en esta región es de $\$ 12.000$ (pesos) en los barcos de los comerciantes del río Putumayo y que el ingreso promedio mensual de estas personas no es superior a los $\$ 40.000$.

Los toldillos se lavan, en promedio, una vez al mes y duran alrededor de dos años. En las visitas realizadas a las viviendas en Arica, se encontraron 189 toldillos y un total de 259 personas, lo que da una cobertura de $73 \%$. Setenta 
toldillos (37\%) presentaban, por lo menos, un agujero de suficiente tamaño para dejar pasar un zancudo.

Otras medidas como el uso de pantalones y camisas de manga larga, la quema de carbón, incienso y caucho también se reconocieron de cierta utilidad. La aspersión doméstica de insecticidas también se mencionó como una medida útil de protección, pero todos coincidieron en que era una medida fuera del alcance de la mayoría debido a su alto costo ( $\$ 3.000$ por mes). Otras actividades de control como el ordenamiento del medio, el relleno de charcos, etc., no fueron contempladas como medidas de control.

Actitudes hacia las actividades de control de malaria organizadas por el Estado: la mayoría reconoce que el trabajo del Grupo de Control de Vectores (malaria) es bueno y ha sido importante para el control de mosquitos. La principal queja de la comunidad es sobre la frecuencia con que se cumplen estas actividades, ya que piensan que se debe hacer como mínimo cada tres meses y, en este momento, hace más de ocho meses que no se realiza fumigación. La comunidad también percibe que el DDT que se usaba anteriormente, daba mejores resultados porque mataba ratas, cucarachas y su efecto residual era mayor. Reconocen actualmente que después de las fumigaciones, se disminuye la actividad general de los mosquitos casi en un $100 \%$.

Conocimiento sobre malaria: en cuanto a los conocimientos sobre malaria, las comunidades estudiadas identifican claramente a la enfermedad clínica como paludismo. El término malaria está reservado para los trabajadores del Grupo de Control de Vectores. Todos identifican los principales síntomas de la malaria, fiebre y escalofríos y que puede ser una enfermedad grave. También mencionan que, antes de la llegada de los blancos a la zona, esta enfermedad no existía. Algunos mencionan que la pérdida de apetito y la falta de ánimos para trabajar son otras características importantes de la malaria. Identifican como signos y síntomas de malaria los que se muestran en la figura 1.

La actividad agrícola juega un papel importante en la zona; de los que se dedican a esta actividad,

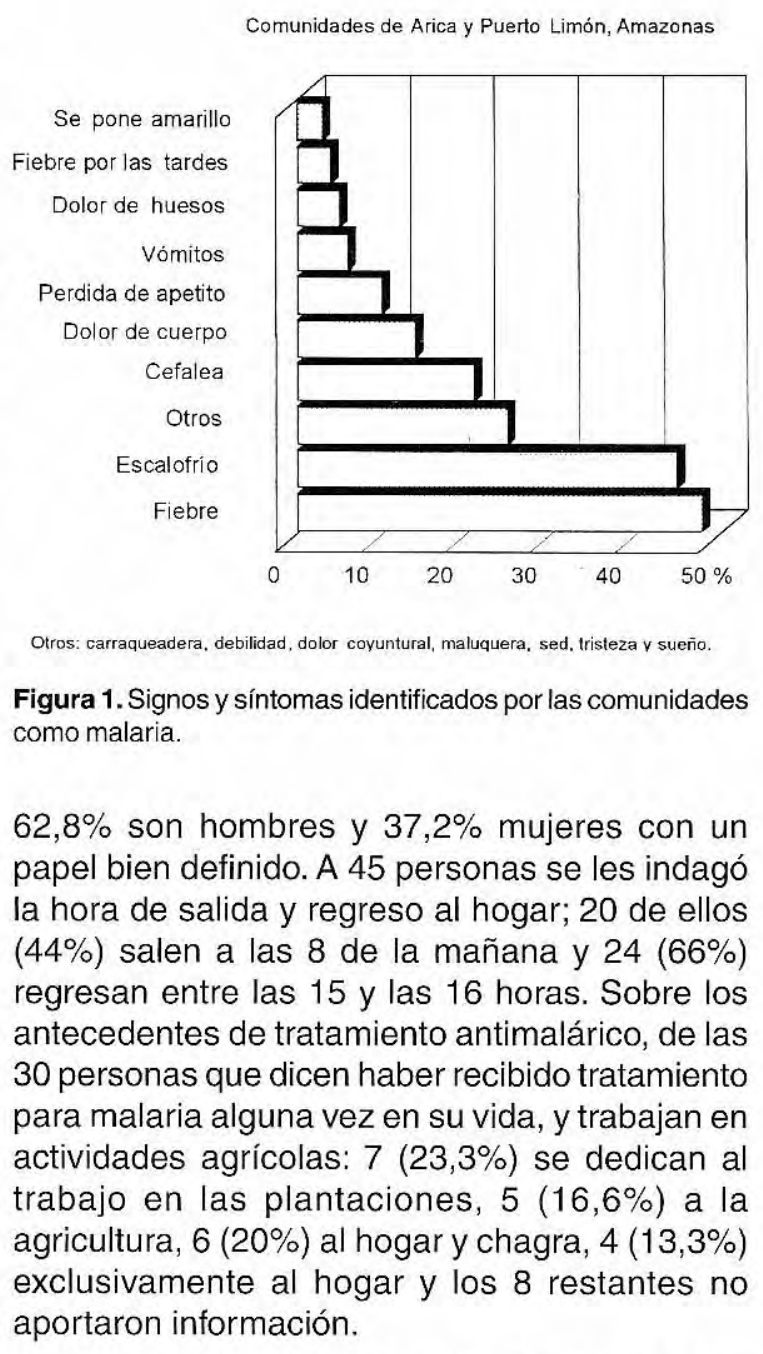

Prevalencia de malaria durante los últimos años: se encuestaron todas las viviendas de Arica, Puerto Limón y Buri Buri. Se obtuvieron datos de 257 personas, la mayoría en Arica (89\%). El promedio de edad de los encuestados era de 22 años (IC 95\%: 20 a 24) y $48 \%$ eran mujeres. Quince personas tenían un cuadro febril en el momento de la encuesta y uno era causado por $P$. vivax $(0,4 \%)$. En el último año, $67(26 \%)$ de las personas habían presentado cuadros febriles; 5 de ellos debidos a malaria (2\%) (IC 95\%: 0,4 a $3,6 \%$ ) confirmados por gota gruesa en el puesto de salud y todos habían recibido tratamiento con cloroquina y sulfa-pirimetamina.

En cuanto a los antecedentes de malaria, 69 $(25 \%)$ de todos los encuestados había tenido 
malaria una vez en su vida; $18 \%$ la última vez que tuvieron malaria fue antes de $1990,27 \%$, entre 1990 y 1994 , mientras que $55 \%$ referían haber tenido un episodio de malaria entre 1995 y el momento de la encuesta. La prevalencia de episodios de malaria aumentaba con la edad, siendo de $9 \%$ en los menores de 15 años (12/ 134), 30\% entre los que tenían de 16 a 24 años (12/40), 40\% entre los de 25 a 44 años (25/63) y $47 \%$ en los mayores de esta edad (18/38).

No hubo diferencias en la prevalencia de malaria por sexo, ni en el análisis crudo, ni estratificado por edad.

Prevalencia de parasitemia en gota gruesa: se tomaron 11 muestras de gota gruesa en Puerto Limón y 26 en Arica. La distribución por edad y sexo era semejante en las dos comunidades. Se encontraron tres personas positivas $(27 \%)$ para trofozoítos de $P$. vivax, entre las muestras tomadas en Puerto Limón, mientras que, en Arica, no hubo ningún positivo $(0 \%)(p<0,05)$. Uno de los pacientes positivos había estado con fiebre durante varios días antes de la muestra y había sido tratado con cloroquina por el promotor de salud, pero, no había recibido primaquina. Los otros dos pacientes positivos eran asintomáticos y fueron tratados con el esquema completo.
Todas las personas positivas para Plasmodium se dedicaban a trabajar en cultivos ilícitos dentro de la selva. Tenían entre 3 y 10 meses de haber llegado a la región y eran hombres entre los 20 y los 35 años. Dentro de las personas a quienes se les tomó muestra en Arica, ninguna se dedicaba a trabajar en cultivos ilícitos dentro de la selva, aunque sí salían a la selva diariamente a trabajar en pequeñas parcelas.

Inspección de criaderos: en la encuesta entomológica, se examinaron 18 criaderos potenciales en Arica, Puerto Limón y Buri Buri. De los diez criaderos examinados en Puerto Limón y Buri Buri, se encontraron formas inmaduras de Anopheles en 2 de ellos que correspondían a chuquios. Todos los criaderos positivos estaban fuera del perímetro habitado de los caseríos. Se capturaron Anopheles adultos en el momento de la inspección de los criaderos en la localidad de Buri Buri, en trampa Shannon en Arica y Puerto Limón, y en cebo humano protegido en el peridomicilio de la vivienda y muy próxima la zona selvática. Se identificaron adultos de Anopheles oswaldoi, Anopheles evansae, Anopheles apicimacula y Aedes fulvus. No se encontraron larvas de Anopheles darlingi (cuadro 1).

No se capturaron especímenes de Aedes aegypti ni de Aedes albopictus. Los promotores de salud

Cuadro 1. Especies de mosquitos identificados por localidad según método de captura.

\begin{tabular}{lll}
\hline Especie (adultos) & Localidad & Método de captura \\
\hline Anopheles oswaldoi & Puerto Limón & Trampa Shannon \\
& Puerto Limón & Trampa CDC \\
Anopheles mediopunctatus & Río Buri Buri & Cebo humano protegido \\
Anopheles apicimacula & Puerto Limón & Trampa Shannon \\
Anopheles evansae & Arica & Trampa Shannon \\
& Puerto Limón & Trampa Shannon \\
Psorophora albipes & Arica & Trampa Shannon \\
& Puerto Limón & Cebo humano protegido \\
Psorofora ferox & Río Buri Buri & Cebo humano protegido \\
& Puerto Limón & Cebo humano protegido \\
Haemagogus janthinomys & Río Buri Buri & Cebo humano protegido \\
Haemagogus anastasionis & Río Buri Buri & Cebo humano protegido \\
Aedes fulvus & Río Buri Buri & Cebo humano protegido \\
Aedes terrens & Río Buri Buri & Cebo humano protegido \\
Mansoniasp. & Río Buri Buri & Cebo humano protegido \\
Culex sp. & Puerto Limón & Cebo humano protegido \\
\hline
\end{tabular}


del área fueron entrenados en las técnicas de búsqueda y captura de mosquitos y larvas.

Comportamiento de la malaria en el departamento: se encontró que en el período de enero de 1996 a abril de 1997, se notificaron 307 casos de malaria en el Departamento del Amazonas. De los casos informados, $72 \%$ eran hombres y $28 \%$ mujeres. La distribución por edad se muestra en el cuadro 2.

Cuadro 2. Distribución de los casos de malaria, por grupos de edad, Amazonas, enero 1996-abril 1997.

\begin{tabular}{lcc}
\hline Edad (años) & Casos & Tasa por mil (IC 95\%) \\
\hline$<5$ años & 17 & $2,5(1,5-4,1)$ \\
5 a 14 & 33 & $2,6(1,8-3,7)$ \\
15 a 24 & 99 & $11,6(9,5-14,0)$ \\
25 a 44 & 108 & $10,5(8,0-17,0)$ \\
45 a 54 & 27 & $12,0(8,0-17,0)$ \\
55 a 64 & 18 & $12,8(8,0-20,6)$ \\
65 y más & 5 & $6,0(2,0-13,0)$ \\
\hline Total & $\mathbf{3 0 7}$ & $7,1(6,3-7,9)$
\end{tabular}

Fuente: Registro ETV, Control de Patologias, Secretaría de Salud del Amazonas, 1997.

Como se observa en este cuadro, la incidencia de malaria se incrementa significativamente después de los 15 años de edad y decrece después de los 65 años con un valor de $p<0,05$.

Se examinó el comportamiento de la malaria en las localidades de Arica, La Chorrera, Buenos
Aires, Araracuara, El Encanto, San José y Tarapacá, por semanas epidemiológicas. Las semanas con mayor actividad de malaria fueron las comprendidas entre la 27 y la 40 . En este período, la mayoría de las localidades notificaron casos de malaria. Tarapacá notificó casos en 38 de las 52 semanas. El cuadro 3 muestra las tasas de infección por corregimiento.

\section{Discusión}

Uno de los principales hallazgos del estudio es la alta prevalencia de parasitemias encontradas en personas que trabajan en cultivos ilícitos dentro del área. La explicación de este hallazgo es que estas personas viven largos períodos de tiempo dentro de la selva, estando más expuestos a las picaduras de los vectores de la malaria que, como lo indica el estudio entomológico, tienen sus criaderos más eficientes dentro del perímetro de las comunidades. Así mismo, trabajan más horas diarias que los indígenas que generalmente trabajan desde las 7 de la mañana hasta las 30 4 de la tarde, mientras que las personas que recogen hojas de coca lo hacen desde las 6 de la mañana hasta las 6 o 7 de la noche, lo que los expone al vector durante las horas vespertinas que es cuando hay mayor actividad de Anopheles. En esta diferencia podría radicar la causa de que los obreros de las plantaciones de coca tengan mayor prevalencia de parasitemia que los habitantes de Arica. Estas personas tienen mayor movilidad y pueden ser amplificadoras de la transmisión de malaria en la región.

Cuadro 3. Distribución de los casos de malaria por corregimiento y tipo de Plasmodium, Amazonas, enero 1996-abril 1997.

\begin{tabular}{lccccc}
\hline Localidad & Casos & $\begin{array}{c}\text { Casos de } \\
\text { P. falciparum }\end{array}$ & $\begin{array}{c}\text { Casos de } \\
\text { P. vivax }\end{array}$ & $\begin{array}{c}\text { Tasa por mil de } \\
\text { P. falciparum }\end{array}$ & $\begin{array}{c}\text { Tasa por mil de } \\
\text { de } P \text {. vivax }\end{array}$ \\
\hline La Pedrera & 7 & 2 & 5 & 1,4 & 3,4 \\
La Chorrera & 13 & 3 & 10 & 1,0 & 3,4 \\
Tarapacá & 75 & 7 & 49 & 3,7 & 26,2 \\
Puerto Santander & 13 & 0 & 10 & 0 & 7,3 \\
Puerto Nariño & 6 & 0 & 6 & 0 & 1,4 \\
El Encanto & 95 & 55 & 31 & 0,3 & 0,12 \\
Leticia & 3 & 0 & 3 & 0 & 1,16 \\
Arica & 3 & 0 & 3 & 2,74 & 0,7 \\
Mirití & 5 & 4 & 1 & 1,4 & 4,4 \\
Puerto Alegría & 4 & 1 & 3 & & \\
\hline
\end{tabular}

Fuente: Registro ETV, Control de Patologías, Secretaría de Salud del Amazonas, 1997 
Los estudios en la comunidad muestran que las personas tienen algunos conocimientos sobre la malaria y la forma como ella se transmite; sin embargo, estos conocimientos son débiles en lo que tiene que ver con la caracterización del vector, dado que ninguno de los entrevistados o encuestados pudo describir adecuadamente el Anopheles. En la comunidad huitota, se encontró que lo conocían y describían muy bien llamándolo virutero, en su idioma, marufaido. Desconocían su papel como transmisor de malaria, posiblemente corresponde al mosquito marudo que mencionaban los pobladores de Arica. Se habla de un mosquito ako que podría corresponder a las especies Psorophora albipes o Psorophora ferox las cuales se encontraron en gran densidad y que alcanzan el domicilio. Ikenakwaje podría corresponder a Aedes fulvus aun cuando este mosquito pica al humano.

En un estudio realizado en Buenaventura, Nieto et al. encontraron que la malaria era considerada un grave problema de salud pública en casi todos los grupos encuestados, mientras que en el presente trabajo, esta sensación de estar amenazados por la malaria no era tan inmediata; la diferencia entre la ocurrencia de malaria en Buenaventura y en el área de nuestro estudio explican el por qué de la diferencia (2). En Buenaventura, hay transmisión de malaria prácticamente todo el año mientras que, en Arica y en las poblaciones encuestadas por nosotros, esta enfermedad solamente ocurre en algunas épocas del año y no siempre con la misma intensidad. Además, en Buenaventura, la mayoría de los casos de malaria es producida por $P$. falciparum que ocasiona la mayoría de las complicaciones. En el Amazonas, el principal causante de malaria es $P$. vivax cuyo cuadro clínico es más benigno. Estas diferencias nos ayudan a corroborar que la forma en que fueron dirigidos los grupos focales no llevaba a respuestas inducidas por parte de los participantes (2).

Por otra parte, en Buenaventura, el conocimiento que la gente tenía sobre la forma de transmisión de la enfermedad era más adecuado que en Arica. En Buenaventura, casi todos los participantes en el estudio sabían cuál era el mecanismo de transmisión de la enfermedad mientras que, en el Amazonas, una proporción importante de personas identificaba mecanismos incorrectos de transmisión. Esta diferencia está influida, muy posiblemente, por la diferencia en los niveles de endemicidad. Además, la exposición de la población a los trabajadores del control de malaria es mucho más fuerte en Buenaventura que en estas poblaciones del río Putumayo donde sólo una vez al año o máximo dos se hacen actividades de fumigación y control.

El conocimiento sobre la sintomatología de la enfermedad es bastante adecuado y un hallazgo importante es el que no existe prácticamente la automedicación en estas áreas. Este hallazgo es contrario a lo encontrado en Buenaventura donde la automedicación es más frecuente y donde, además, se usan también métodos de la medicina tradicional para combatir la enfermedad. La automedicación puede llevar a formas severas de malaria y al enmascaramiento de los casos clínicos con el consiguiente riesgo de que las personas parasitémicas sin tratamiento, lleven la enfermedad a otras localidades donde no existe la enfermedad, pero sí el vector.

La principal preocupación de la comunidad con respecto a los mosquitos no es la de que transmitan enfermedades potencialmente mortales como la malaria o la fiebre amarilla. La principal motivación de la comunidad para controlar estos insectos es debido a las molestias que producen por su picadura. En este sentido, un programa de control de vectores debería tener objetivos más amplios que los de solamente combatir vectores de importancia médica, para lograr la cooperación entusiasta de la comunidad. Es posible que en estas comunidades, se deban alternar las fumigaciones con los programas de control de ratas. Perciben, también, que los barcos que transitan por el río con bastante frecuencia, pueden ser diseminadores de mosquitos y de otras plagas; por tanto, sería necesario tratar de realizar un buen control sanitario en esas embarcaciones para reducir la infestación.

Contrario a lo hallado en otros países endémicos para malaria, la proporción de personas que se 
autoprotegen con toldillos es muy alta en esta zona del Amazonas $(3,4)$. A pesar del alto costo del toldillo, comparado con el ingreso promedio de las personas de la región, todas las personas en las viviendas visitadas tenían toldillo. Un porcentaje importante de los toldillos revisados tenía perforaciones lo que disminuye su eficacia.

Es importante educar a la gente sobre la necesidad de no fumar dentro del toldillo y de reparar los daños que sufra. Esta alta prevalencia de toldillos hace posible la estrategia de controlar la malaria al impregnarlos con piretroides, lo cual asegura una buena educación a las personas que deben lavar los toldillos con un intervalo no inferior a los tres meses y no cada 15 días como se acostumbra. El personal de salud debe hacer evaluaciones periódicas para que estas instrucciones se lleven a cabo.

La mayoría de las personas entrevistadas considera que las actividades de control del Estado son buenas, aunque se quejan de la falta de efecto residual del producto que se emplea actualmente para las fumigaciones que es la lambdacihalotrina. El éxito del Programa de Control de Vectores depende también de la satisfacción que las personas sientan con su ambiente, así que es probable que una disminución del intervalo de tiempo entre las aspersiones haga que la comunidad se sienta más satisfecha.

La evaluación entomológica preliminar revela que los criaderos de anofelinos encontrados están localizados en la selva, algunos de ellos cerca de la vivienda $(100-500 \mathrm{~m})$. Los criaderos encontrados son temporales, como consecuencia de la alta precipitación que se estaba presentando en el momento de realizar esta investigación. Lo más probable es que existan más criaderos temporales y permanentes selva adentro; los colonos e indígenas mencionan la existencia de las lagunas dentro de la selva donde acostumbran ir a pescar, lo que los hace susceptible a infectarse por malaria en estos lugares. Este hecho como también el que se desplazan a las selvas a atender sus chagras sin la debida protección, reforzaría la hipótesis en el sentido de que la mayor transmisión se estaría dando dentro de la selva, en donde se están desarrollando los anofelinos. Sin embargo, algunos mosquitos también pueden llegar al domicilio (peri e intra); se tienen registros de dispersión de anofelinos menores a $1 \mathrm{~km}$ y mayores de $15 \mathrm{~km}$ (bajo número).

Resultó sorprendente no encontrar la especie $A$. darlingiconsiderado el vector de malaria para esta región del país (1). Es posible que debido a la alta precipitación pluvial que se presentaba en esta época, se haya reducido la población, caso similar a lo encontrado en ciertas áreas de los Llanos Orientales (V.A. Olano, comunicación personal). Sin embargo, se registró la especie $A$. oswaldoi transmisora de Plasmodium en los estados brasileños de la Amazonia y otros países (5-9).

Los criaderos encontrados positivos para anofelinos se encuentran dentro de la selva; no es viable pensar en un control larvario realizando intervenciones dirigidas al control físico como es el ordenamiento del medio. Se encontraron criaderos potenciales para Anopheles en áreas urbanas y periurbanas.

Un estudio entomológico más amplio y en época lluviosa y seca, permitiría obtener datos concluyentes sobre las especies de Anopheles vectoras de la malaria y sus lugares de cría en esta región del Amazonas.

El presente estudio revela que la malaria en el Departamento del Amazonas es de bajo riesgo en la mayoría de las zonas por tener un índice parasitario anual (IPA) menor de 5 por 1000 habitantes. La localidad que presenta más casos durante todo el año es Tarapacá, donde se registra una fuerte actividad de explotación maderera. Dadas las tasas de ataque de malaria por edad, podemos suponer que la mayor parte de la transmisión es de tipo ocupacional, es decir, personas que en su oficio deben internarse a la selva para trabajar sin la protección adecuada y que están expuestas a las picaduras de mosquitos infectados con malaria.

Los casos de mujeres podrían explicarse por la transmisión intradomiciliaria; sin embargo, también la mujer es fuerza de trabajo importante 
en la Amazonia y dentro de sus funciones hogareñas está la de atender la chagra casi diariamente; por tanto, creemos que la mayoría de los casos en mujeres es debida a la exposición fuera del domicilio.

Con base en los resultados del estudio, se hicieron las siguientes recomendaciones a la Secretaría de Salud del Amazonas:

1) Reforzar la vigilancia epidemiológica en las áreas del departamento que más reportan casos, como Tarapacá y Arica. Esta vigilancia podría volverse más activa aprovechando que hay un cuerpo de promotores de salud indígenas que han sido entrenados en la toma de gota gruesa con muy buenos resultados de concordancia. Esta experiencia de la zona de La Chorrera y Arica con promotores sería de gran utilidad si se ampliara al corregimiento de Tarapacá, dotándolos con la respectiva capacitación de los equipos de radiocomunicaciones, microscopios y combustible. Los promotores podrían visitar periódicamente los campamentos de madereros y realizar la toma de gotas gruesas tanto en pacientes febriles como en asintomáticos y suministrar el tratamiento a los positivos. Esto probablemente disminuirá la frecuencia de portadores de parásitos y, por ende, las fuentes de infección para el vector.

2) Añadir algunas variables al formulario de notificación de enfermedades transmitidas por vectores -entre ellas, malaria-, registro unificado que se maneja actualmente, que podrían ayudar a identificar mejor la procedencia y la actividad económica a que se dedican los casos.

3) Fomentar la coordinación intersectorial con la gobernación del Amazonas y con el Cabildo Indígena para que, en los programas de mejoramiento de vivienda en Arica ya sea con recursos de transferencia o por otros tipos de proyectos de inversión social, se incluya el suministro de anjeos; se observó que la tendencia de la construcción de las nuevas viviendas es a usar el anjeo, a pesar de las fuertes limitaciones económicas.
4) Desarrollar programas de tipo integral e integrado que involucren a la comunidad e incentiven la conformación de la red entomológica con los promotores de salud. Fomentar el uso de toldillos impregnados, de ropa cubierta y control químico según las recomendaciones del Ministerio de Salud y siguiendo la metodología de aplicación.

5) Desarrollar programas de saneamiento, los cuales incluyen el control de insectos y la eliminación de roedores; la fumigación de los barcos comerciantes que viajan por el río Putumayo evitaría también el ingreso de $A$. aegypti a esta zona.

\section{Referencias}

1. Ministerio de Salud de Colombia. Situación epidemiológica de la malaria en Colombia. Informe del Programa de Control de Vectores, 1996.

2. Nieto T, Méndez F, Carrasquilla G. Knowledge, beliefs and practices relevant for malaria control in an endemic urban area of the Colombian Pacific. Informe final. FES, 1996.

3. Baird J, Sismadi P, Masbar S, Ramzan A, Purnomo B , Sekartuti C, et al. A focus of endemic malaria in central Java. Am JTrop Med Hyg 1996;54:98-104.

4. Klein R, Weller S, Zeissig R, Richards F, Ruebush T. Knowledge, beliefs, and practices in relation to malaria transmission and vector control in Guatemala. Am J Trop Med Hyg 1995;52:383-8.

5. Klein T, Lima J, Tada M. Comparative susceptibility of anopheline mosquitoes to Plasmodium falciparum in Rondonia, Brazil. Am J Trop Med Hyg 1991;44:598-603.

6. Klein T, Lima J, Tada M, Miller R. Comparative susceptibility of anopheline mosquitoes in Rondonia, Brasil to infection by Plasmodium vivax. Am J Trop Med Hyg 1991;45:463-70.

7. Deane L. Chronology of the discovery of malaria vectors in the Brazilian Amazon. Mem Inst Oswaldo Cruz 1989;84(Supp. IV):149-56.

8. Branquinho M, Araujo M, Natal D, Marrelli M, Rocha R, Taveira F, Kloetzel J. Anopheles oswaldoi a potential malaria vector in Acre, Brazil. Trans Roy Soc Trop Med Hyg 1996;90:233.

9. Branquinho M, Lagos C, Rocha R, Natal D, Barata J, Cochrane A, et al. Anophelines in the state of Acre, Brazil, infected with Plasmodium falciparum, P. vivax, the variant $P$. vivax VK247 and $P$. malarie. Trans Roy Soc Trop Med Hyg 1993;87:391-4. 